\title{
Efeito Residual de Flumioxazin sobre a Emergência de Plantas Daninhas em Solos De TeXturas Distintas ${ }^{1}$
}

\author{
Residual Effect of Flumioxazin on Weed Emergence in Soils of Distinct Textures \\ JAREMTCHUK, C.C..$^{2}$, CONSTANTIN, J. ${ }^{3}$, OLIVEIRA JÚNIOR, R.S. ${ }^{3}$, ALONSO, D.G..$^{4}$, \\ ARANTES, J.G.Z. ${ }^{4}$, BIFFE, D.F. ${ }^{4}$, ROSO, A.C. ${ }^{5}$ e CAVALIERI, S.D. ${ }^{6}$
}

\begin{abstract}
RESUMO - Com o objetivo de avaliar o efeito residual de flumioxazin sobre a emergência de plantas daninhas em solos de texturas distintas, foi realizado um experimento em casa de vegetação. Os tratamentos foram constituidos pela combinação de quatro épocas de aplicação: 14, 10, 7 e 0 dias antes da semeadura das espécies de plantas daninhas, com duas doses de flumioxazin (25 e $40 \mathrm{~g} \mathrm{ha}^{-1}$ ) e uma testemunha sem aplicação para cada planta daninha em solos de texturas distintas. O efeito residual do herbicida flumioxazin foi avaliado por meio da contagem do número de plantas emersas de cada espécie aos 35 dias depois da semeadura (DDS). O controle do fluxo inicial de emergência de plantas daninhas variou em função do tipo de solo, do periodo de tempo entre a aplicação e a semeadura das espécies e também da dose. A. tenella, D. horizontalis, D. insularis, D. tortuosum, E. heterophylla, $N$. physaloides e S. latifolia foram as espécies mais sensiveis à aplicação de flumioxazin (25 e $\left.40 \mathrm{~g} \mathrm{ha}^{-1}\right)$.
\end{abstract}

Palavras-chave: herbicida, atividade residual, controle de plantas daninhas.

\begin{abstract}
This work aimed to evaluate the residual effect of flumioxazin on weed emergence in soils of distinct textures. Treatments were constituted by the combination of four periods of application (14, 10, 7 and $O$ days before sowing) with two rates of flumioxazin (25 and $40 \mathrm{~g}$ ha$\left.{ }^{1}\right)$, and no herbicide application for each weed species in soils with distinct textures. Residual activity of flumioxazin was evaluated by counting the emerged weeds for each species at 35 days after sowing. Control of initial flows of weed emergence varied with soil type, period of time between herbicide application and weed sowing. A. tenella, D. horizontalis, D. insularis, D. tortuosum, E. heterophylla, N. physaloides and S. latifolia, in both soils, were the most susceptible species to flumioxazin, applied at 25 and $40 \mathrm{~g} \mathrm{ha}$.
\end{abstract}

Keywords: herbicide, residual activity, weed control.

\section{INTRODUÇÃO}

Flumioxazin é um herbicida registrado para uso em condições de pré-emergência, no controle de plantas daninhas de folhas largas e de algumas monocotiledôneas na cultura da soja (Oliveira et al., 1998). Por ser utilizado em pré-emergência, é fundamental que se conheça sua atividade no solo. O conhecimento dos processos de sorção e lixiviação dos herbicidas no solo permite a recomendação de doses diferenciadas por tipo de solo e, consequentemente, o controle mais eficiente das plantas daninhas (Lima et al., 1999).

Recebido para publicação em 23.2.2008 e na forma revisada em 18.2.2009.

2 Enga - Agr ${ }^{\mathrm{a}}$, M.Sc., <carlacjk@yahoo.com.br>; ${ }^{3}$ Professor Associado, Núcleo de Estudos Avançados em Ciência das Plantas Daninhas (NAPD/UEM), Dep. de Agronomia, Universidade Estadual de Maringá; ${ }^{4}$ Engō-Agro ${ }^{\circ}$ Mestrando do Programa de PósGraduação em Agronomia, NAPD/UEM; ${ }^{5}$ Eng - aggr ${ }^{\mathbf{a}}$, Mestranda do Programa de Pós-Graduação em Agronomia, UFRGS; ${ }^{6}$ Engo Agr ${ }^{-}$, Doutorando do Programa de Pós-Graduação em Agronomia, FCA/UNESP. 
Segundo Rodrigues \& Almeida (2005), o flumioxazin é adsorvido pelos colóides do solo, com lixiviação reduzida. Em razão de se caracterizar como uma molécula não dissociável (Hatzios, 1998), o pH do solo pouco afeta seu comportamento. Apresenta rápida dissipação no solo (meia-vida de 11,9 a 17,5 dias) e eficácia sobre biótipos de plantas daninhas resistentes aos herbicidas inibidores de ALS e triazinas (Taylor-Lovell et al., 2001).

Estudos demonstram que o teor de matéria orgânica nos diferentes tipos de solo influencia a adsorção do flumioxazin, indicando que as doses desse herbicida devem ser recomendadas de acordo com a capacidade adsortiva dos solos (Oliveira et al., 1998; Lima et al., 1999).

Em razão da diversidade de espécies de plantas daninhas e da dificuldade em avaliar niveis de controle, a atividade de herbicidas de pré-emergência pode ser mais bem avaliada em vasos. Dessa forma, permite-se verificar o efeito residual do herbicida para cada espécie de planta daninha isoladamente.

As infestações de plantas daninhas são normalmente desuniformes, o que implica alta variabilidade dos dados e compromete a análise dos resultados. Niveis de controle de 40 a $60 \%$, por exemplo, podem ser considerados não-significativos. Contudo, se fossem considerados como benefício adicional da dessecação com flumioxazin, passariam a ter grande importância, sem a ocorrência da interferência precoce, que pode também flexibilizar a aplicação de herbicidas de pós-emergência. Quanto ao flumioxazin, detectou-se eficácia no controle de $A$. viridis, $B$. pilosa, I. grandifolia e $P$. oleracea, com $25 \mathrm{~g}^{-1}$, e de $A$. hispidum e $D$. horizontalis, com doses superiores, por até 28 dias após a aplicação, na cultura da cebola (Rozanski et al., 2002). Também nessa cultura, Durigan et al. (2005) obtiveram excelente controle de $L$. virginicum, até 80 dias após a aplicação, em todas as doses avaliadas (de 80 a $240 \mathrm{~g} \mathrm{ha}^{-1}$ ). Burke et al. (2002) constataram que o flumioxazin (71 e $105 \mathrm{~g} \mathrm{ha}^{-1}$ ) controlou $89 \%$ de $S$. spinosa. Scott et al. (2001) constataram que o controle de $C$. album e I. hederacea com flumioxazin (87 $\left.\mathrm{g} \mathrm{ha}^{-1}\right)$ aplicado em préemergência foi eficiente. Niekamp et al. (1999) verificaram que o flumioxazin (90 $\left.\mathrm{g} \mathrm{ha}^{-1}\right)$ promoveu o controle de $92 \%$ de $A$. theophrasti e $A$. artemisiiofia, $89 \%$ de $X$. strumarium e $96 \%$ de I. lacunosa. Askew et al. (1999) verificaram que o flumioxazin (70 e $110 \mathrm{~g} \mathrm{ha}^{-1}$ ) controlou $96 \%$ de $S$. spinosa. No entanto, há poucas informações disponiveis relacionadas à eficácia do flumioxazin em relação a plantas daninhas importantes na agricultura tropical.

O objetivo deste trabalho foi avaliar o efeito residual de flumioxazin no controle inicial de algumas espécies de plantas daninhas em solos de texturas distintas.

\section{MATERIAL E MÉTODOS}

O ensaio foi conduzido no primeiro semestre do ano de 2005, em casa de vegetação da Universidade Estadual de Maringá, em Maringá, Paraná. Como substrato, foram utilizados solos de texturas distintas com a seguinte composição: a) textura de solo arenoso: areia $(70 \%)$, argila (14\%) e silte (16\%); e b) textura de solo argiloso: argila (83\%), areia (15\%) e silte $(2 \%)$. O solo foi seco, peneirado e colocado em vasos com capacidade para $5 \mathrm{dm}^{3}$, os quais foram considerados como unidades experimentais. A análise de solo revelou as seguintes características químicas: a) textura de solo arenoso: $\mathrm{pH}$ água: 5,8; $\mathrm{P}: 9,4 \mathrm{mg} \mathrm{dm}^{-3}$; $\mathrm{K}$ : $0,06 \mathrm{cmol}_{\mathrm{c}} \mathrm{dm}^{-3} ; \mathrm{Al}^{+3}: 0,0 \mathrm{cmol}_{\mathrm{c}} \mathrm{dm}^{-3} ; \mathrm{H}+\mathrm{Al}$ : $2,73 \mathrm{cmol}_{\mathrm{c}} \mathrm{dm}^{-3} ; \mathrm{Ca}^{+2}: 1,73 \mathrm{cmol}_{\mathrm{c}} \mathrm{dm}^{-3} ; \mathrm{Mg}^{+2}$ : $0,87 \mathrm{cmol}_{\mathrm{c}} \mathrm{dm}^{-3}$; C: $7,58 \mathrm{~g} \mathrm{dm}^{-3} ; \mathrm{V}: 49,00 \%$; CTC: $5,39 \%$; e b) textura de solo argiloso: $\mathrm{pH}$ água: 7,8; P: 3,0 $\mathrm{mg} \mathrm{dm}^{-3}$; $\mathrm{K}: 0,51 \mathrm{cmol}_{\mathrm{c}} \mathrm{dm}^{-3}$; $\mathrm{Al}^{+3}: 0,0 \mathrm{cmol}_{\mathrm{c}} \mathrm{dm}^{-3} ; \mathrm{H}+\mathrm{Al}: 1,88 \mathrm{cmol}_{\mathrm{c}} \mathrm{dm}^{-3}$; $\mathrm{Ca}^{+2}:$ 9,70 $\mathrm{cmol}_{\mathrm{c}} \mathrm{dm}^{-3} ; \mathrm{Mg}^{+2}: 1,87 \mathrm{cmol}_{\mathrm{c}} \mathrm{dm}^{-3}$; C: $11,17 \mathrm{~g} \mathrm{dm}^{-3}$; V: $86,53 \%$; CTC: $13,96 \%$. O delineamento experimental utilizado foi o de blocos ao acaso com quatro repetições, em esquema fatorial $(4 \times 2)+1$. Para cada experimento, os tratamentos foram constituídos pela combinação de quatro períodos de aplicação $(14,10,7$ e 0 dias antes da semeadura das espécies de plantas daninhas) com doses de flumioxazin (25 e $40 \mathrm{~g} \mathrm{ha}^{-1}$ ) mais uma testemunha sem aplicação.

As semeaduras foram realizadas no dia 25/2/2005. As espécies de plantas daninhas Alternanthera tenella, Bidens pilosa, Brachiaria decumbens, Brachiaria plantaginea, Cenchrus echinatus, Commelina benghalensis, 
Desmodium tortuosum, Digitaria horizontalis, Digitaria insularis, Euphorbia heterophylla, Ipomoea grandifolia, Nicandra physaloides, Pennisetum glaucum, Richardia brasiliensis, Sida rhombifolia, Spermacoce latifolia e Tridax procumbens foram semeadas $(1-2 \mathrm{~cm}$ de profundidade) em cada repetição. O efeito residual do herbicida flumioxazin foi avaliado por meio da contagem do número de plantas emersas de cada espécie, aos 35 dias depois da semeadura(DDS).

O flumioxazin foi aplicado usando-se um pulverizador, pressurizado com $\mathrm{CO}_{2}$, provido de barra com três pontas de jato leque, AD 110.02, espaçados entre si de $0,5 \mathrm{~m}$, calibrado para aplicação de $200 \mathrm{~L} \mathrm{ha}^{-1}$ e pressão de aspersão de 3,0 $\mathrm{kgf} \mathrm{cm}^{-2}$. Os vasos foram mantidos livres de outras plantas daninhas e irrigados diariamente.

A análise estatística foi efetuada seguindo o modelo tradicional de fatorial com testemunha adicional (Gomes, 1990). Os dados analisados foram transformados em $\log (x+1)$, e as médias apresentadas são referentes aos dados originais. Na presença da interação significativa $(P<0,05)$, procedeu-se aos desdobramentos necessários. Para verificar o efeito dos períodos de aplicação dentro de cada dose, empregou-se o teste de Tukey. Para comparação destes em relação à testemunha, utilizou-se o teste de Dunnett a $5 \%$ de probabilidade.

\section{RESULTADOS E DISCUSSÃO}

O efeito residual no controle do fluxo inicial de emergência de plantas daninhas variou em função do tipo de solo, do período de tempo entre a aplicação e a semeadura das espécies e também da dose (Tabelas 1 e 2).

Em solo argiloso, para as doses de 25 e $40 \mathrm{~g} \mathrm{ha}^{-1}$, as maiores reduções no número de plantas de $T$. procumbens, S. latifolia, $D$. tortuosum, E. heterophylla e D. horizontalis ocorreram no período de aplicação de O DAS. Para A. tenella as reduções foram maiores tanto para a aplicação a 0 como para aplicação aos 7 DAS, o que revela maior sensibilidade desta espécie a este herbicida. Para as espécies $R$. brasiliensis, I. grandifolia e $C$. benghalensis, o flumioxazin reduziu a emergência das plantas apenas na maior dose $\left(40 \mathrm{~g} \mathrm{ha}^{-1}\right)$ e para aplicação a 0 DAS. Segundo Oliveira et al. (1999), a aplicação de $60 \mathrm{~g}^{\text {ha }}{ }^{-1}$ de flumioxazin em Latossolo Roxo distrófico promoveu controle de $C$. benghalensis. Han et al. (2002) verificaram que o flumioxazin (75 $\mathrm{g} \mathrm{ha}^{1}$ ) manteve controle considerável sobre algumas gramineas, como $D$. sanguinalis e E. crus-gali, em solo com $\mathrm{pH} 7,5$ e $1,5 \%$ de matéria orgânica. Wilson et al. (2002) também verificaram que o flumioxazin (35 e $70 \mathrm{~g} \mathrm{ha}^{-1}$ ) promoveu excelente controle de $C$. album e A. retroflexus.

Em solo arenoso, para as doses de 25 e $40 \mathrm{~g} \mathrm{ha}^{-1}$, as maiores reduções no número de plantas de S. latifolia, E. heterophylla, $S$. rhombifolia, $D$. horizontalis e $B$. plantaginea ocorreram nos períodos de aplicação a 0 e 7 DAS; para B. decumbens, as reduções foram observadas até o período de aplicação de 10 DAS. Quanto às espécies B. pilosa, $A$. tenella e $P$. glaucum, o herbicida reduziu a emergência de plantas apenas no período de aplicação de 0 DAS.

A atividade do flumioxazin pode variar em função da adsorção ao solo, sendo esta a possível explicação para a variação de controle das plantas daninhas observada entre os períodos de aplicação e doses. Estudos demonstram que o teor de matéria orgânica nos diferentes tipos de solo influencia a adsorção do flumioxazin, indicando que as doses desse herbicida devem ser recomendadas de acordo com a capacidade adsortiva dos solos (Oliveira et al., 1998; Lima et al., 1999).

Em solo argiloso, pequenas diferenças de controle entre as doses foram observadas para o período de aplicação de 0 DAS. Em solo arenoso, a utilização de $25 \mathrm{~g} \mathrm{ha}^{-1}$ de flumioxazin apresentou controle na maioria das espécies. Ao comparar a atividade de flumioxazin nos solos, foi observado que no arenoso o herbicida apresentou controle das espécies de plantas daninhas mesmo quando aplicado com maior antecipação em relação à semeadura destas. Em estudos desenvolvidos em colunas de solo, não se encontrou nenhum resíduo do herbicida em profundidade maior que $7,6 \mathrm{~cm}$, indicando baixo potencial de lixiviação e rápida degradação no solo (Hazardous..., 2006).

Planta Daninha, Viçosa-MG, v. 27, n. 1, p. 191-196, 2009 
Tabela 1 - Número de plantas emersas por vaso em solos argiloso e arenoso, aos 35 DDS, após quatro períodos de aplicação (0,7,10 e 14 DAS), com doses de flumioxazin ( 25 e $40 \mathrm{~g} \mathrm{ha}^{-1}$ ) para espécies de plantas daninhas dicotiledôneas

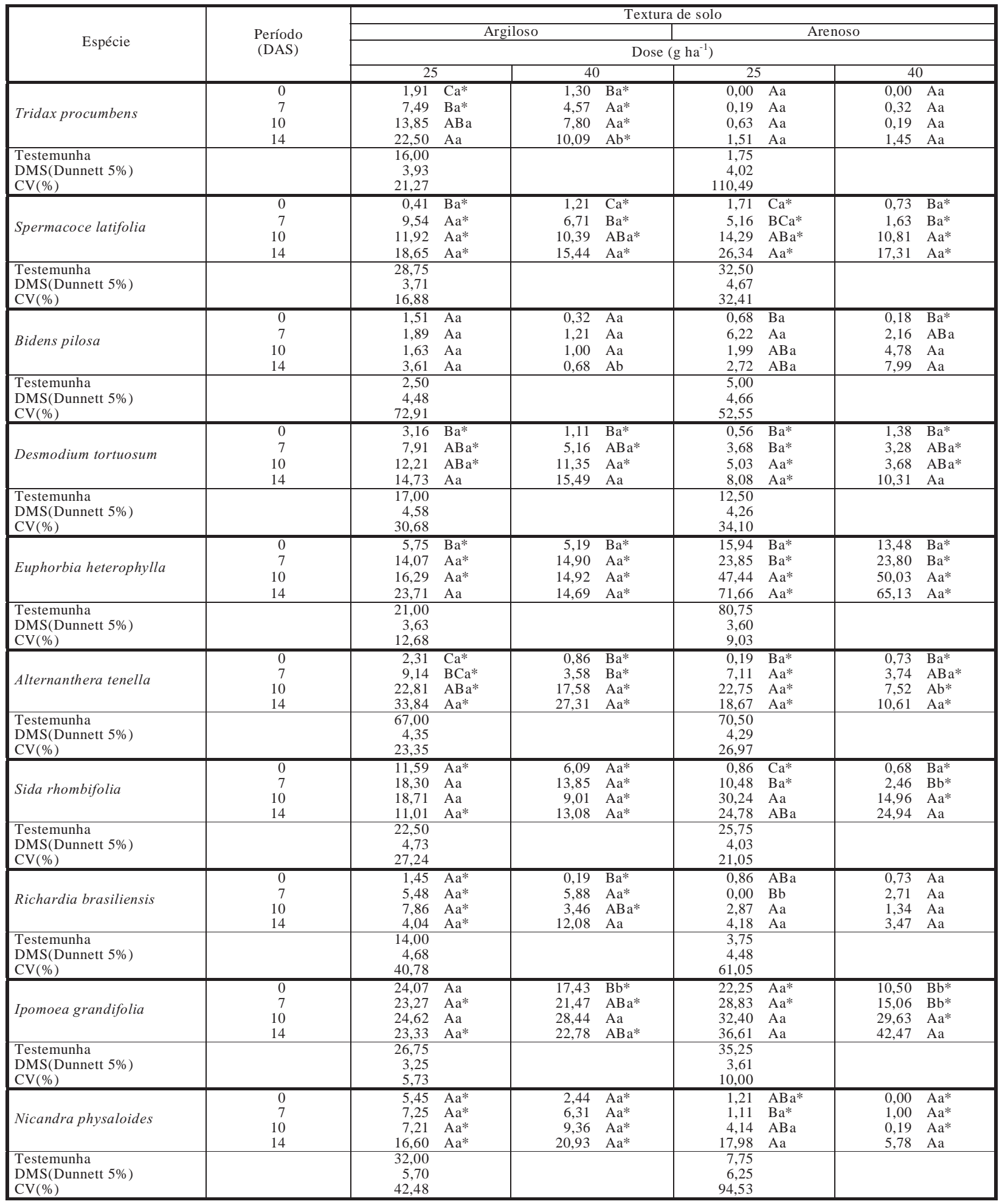

Médias seguidas de mesma letra, maiúscula na coluna ou minúscula na linha, não diferem pelo teste de Tukey a 5\%. Médias seguidas por * foram inferiores à testemunha pelo teste de Dunnett a $5 \%$.

DDS: dias depois da semeadura; e DAS: dias antes da semeadura. 
Efeito residual de flumioxazin sobre a emergência de plantas ...

Tabela 2 - Número de plantas emersas por vaso em solos argiloso e arenoso, aos 35 DDS, após quatro períodos de aplicação $(0,7,10$ e 14 DAS), com doses de flumioxazin ( 25 e $40 \mathrm{~g} \mathrm{ha}^{-1}$ ) para espécies de plantas daninhas monocotiledôneas

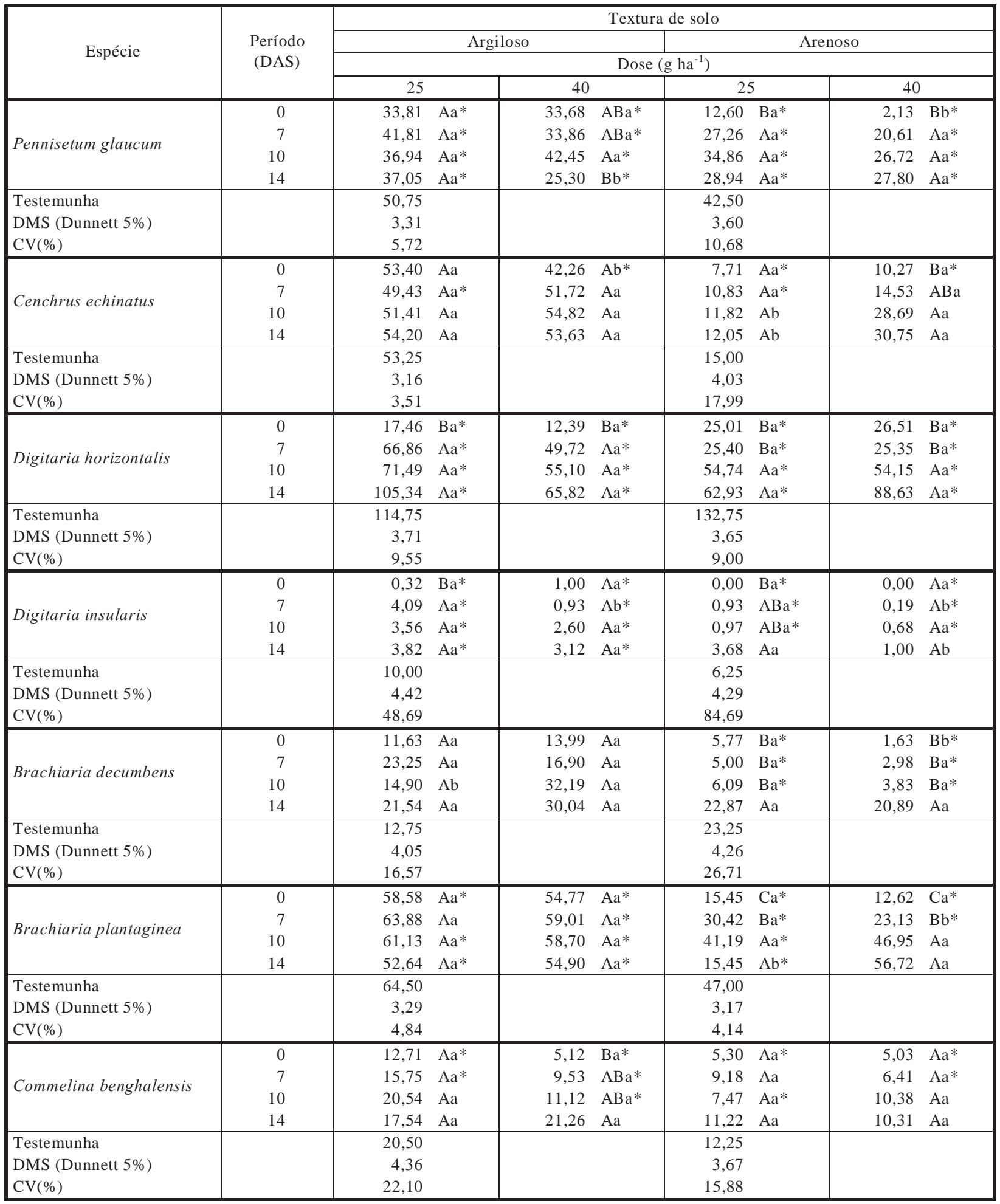

Médias seguidas de mesma letra, maiúscula na coluna ou minúscula na linha, não diferem pelo teste de Tukey a 5\%. Médias seguidas por * foram inferiores à testemunha pelo teste de Dunnett a $5 \%$.

DDS: dias depois da semeadura; e DAS: dias antes da semeadura. 
As espécies daninhas $B$. decumbens, $B$. plantaginea e $P$. glaucum apresentaram controle apenas em solo arenoso. $T$. procumbens e $R$. brasiliensis mostraram controle apenas em solo argiloso. Com base nos resultados deste experimento (Tabelas 1 e 2), podese concluir que $A$. tenella, $D$. horizontalis, $D$. insularis, D. tortuosum, E. heterophylla, N. physaloides e $S$. latifolia foram as espécies mais sensiveis à aplicação de flumioxazin (25 e $40 \mathrm{~g} \mathrm{ha}^{-1}$ ).

\section{AGRADECIMENTOS}

A Luiz Machado Homem, Milton Lopes da Silva e alunos de graduação do Centro de Ciências Agrárias da Universidade Estadual de Maringá, que, com sua participação, tornaram possivel a realização deste trabalho.

\section{LITERATURA CITADA}

ASKEW, S. D.; WILCUT, J. W.; CRANMER, J. R. Weed management in peanut (Arachis hypogea) with flumioxazin preemergence. Weed Technol., v. 13, n. 3, p. 594-598, 1999.

BURKE, I. C.; ASKEW, S. D.; WILCUT, J. W. Flumioxazin systems for weed management in North Carolina Peanut (Arachis hypogaea). Weed Technol., v. 16, n. 4, p. 743-748, 2002.

DURIGAN, J. C.; SILVA, M. R. M.; AZANIA, A. A. P. M. Eficácia e seletividade do herbicida flumioxazin aplicado em pré-emergência na cultura transplantada da cebola. R. Bras. Herbic., v. 4, n. 3, p. 11-17, 2005.

GOMES, F. P. Curso de estatística experimental. 13.ed. Piracicaba: Nobel, 1990. 468 p.

HAN, J. et al. Weed control in summer-sown soybeans with flumioxazin plus acetochlor and flumiclorac-pentyl plus clethodim. Weed Biol. Manag., v. 2, n. 2, p. 120-122, 2002

HATZIOS, K. K. Herbicide handbook. 7.ed. Champaign: Weed Science Society of America, 1998. 104 p.
HAZARDOUS SUBSTANCES DATA BANK.

Flumioxazin. Disponível em: <www.fluoridealert.org/ pesticides/flumioxazin.hsdb.htm>. Acesso em: 20 jan 2006.

LIMA, R. O. et al. Comportamento do herbicida flumioxazin em solo com diferentes doses de calcário. R. Ceres, v. 46, n. 268 , p. $607-613,1999$

NIEKAMP, J. W.; JOHNSON, W. G.; SMEDA, R. J. Broadleaf weed control with sulfentrazone and flumioxazin in no tillage soybean (Glycine max). Weed Technol., v. 13, n. 2, p. 233-238, 1999.

OLIVEIRA, M. F. et al. Lixiviação de flumioxazin e metribuzin em dois solos em condições de laboratório. Planta Daninha, v. 17, n. 2, p. 207-214, 1999

OLIVEIRA, M. F.; SILVA, A. A.; NEVES, J. C. L. Influência do tamanho do agregado e do nível de umidade do solo na atividade do flumioxazin. R. Ceres, v. 45, n. 257, p. 81-87, 1998.

RODRIGUES, B. N.; ALMEIDA, F. S. Guia de herbicidas. 5.ed. Londrina: Edição dos Autores, 2005.

ROZANSKI, A et al. Efeito do herbicida flumioxazin nas plantas daninhas e na cultura da cebola. Disponível em $<$ http://www.abhorticultura.com.br/biblioteca/Default.asp?id= 3284>. Acesso em: 28 maio 2008

SCOTT, G. H.; SHAWN, D. A.; WILCUT, J. W. Economic evaluation of diclosulam and flumioxazin systems in peanut (Arachis hypogaea). Weed Technol., v. 15, n. 2, p. 360-364, 2001.

TAYLOR-LOVELL, S.; WAX, L. M.; NELSON, R. Phytotoxic response and yield of soybean (Glycine max) varieties treated with sulfentrazone or flumioxazin. Weed Technol., v. 5, n. 1, p. 5-102, 2001

WILSON, D. E.; NISSEN, S. J.; THOMPSON, A. Potato (Solanum tuberosum) variety and weed response to sulfentrazone and flumioxazin. Weed Technol., v. 16, n. 3, p. $567-574,2002$. 